\title{
Histologische Untersuchungen über die Haut der Mischlinge.
}

\author{
Von \\ Tetsuo Watanabe. \\ Aus dem Anatomischen Institut der Keio Universität, Tokyo \\ (Director: Prof. Dr. T. Taniguchi).
}

Mit 2 Textfiguren.

\section{Einleitung.}

Über das Hautpigment der japanischen Feten sind bisher zahlreiche Forschungen erschienen. So finden wir zuerst die histologische Untersuchung über das Hautpigment von Bälz (1885), später dieselbe von Grimm (1895). Beide Forscher wiesen damals in ihrer gemeinsamen Untersuchung über das sogenannte Mongolenfleck darauf hin, $\mathrm{da} \beta$ dasselbe der mongoloiden Rasse eigentümlich sei. Spätere Autoren, wie z. B. Muto und Miwa (1924) beschäftigten sich in ihrer genetischen Untersuchung des Pigments an der Haut des Kopfes und Rückens von 32 Feten, Morooka, Hasegawa, Iizuka und Kojima in derselben an der Haut der verschiedenen Körperteile. Hinsichtlich der japanischen Feten sind die wichtigen Arbeiten von Adachi (1903) und Bahrawy (1922) anzuführen.

Kudo berichtete neuerdings (1947) in seiner histologischen Untersuchung über je einen Mischling von Japanerin und Europäer und Neger, da $B$ wischen ihnen und den zur Kontrolle herangezogenen japanischen Feten ein merklicher Unterschied nicht besteht. Ich selbst habe das Hautpigment der Mischlinge histologisch untersucht, und darf hier über die Erkenntnis berichten.

\section{Material und Methode.}

Das Material erfasste 8 Mischlingsfeten $z$ wischen Japanerinnen und Europäern vom 4 . bis 8 . Fetalleben, 1 achtmonatigen zwischen Japanerin und Neger und 1 Mischlingsneugeborenen (welcher 2 Tage nach der 
Geburt lebte) $z$ wischen Japanerin und Europäer, im ganzen 10 Mischlingsfeten. Zwecks Vergleich wurden 9 japanische Feten von gleichem Geschlecht, Alter und fast gleicher Körpergröße herangezogen, indem sich die Gesamtzahl auf 19 Feten erhöhte.

Tabelle 1. Untersuchungsmaterial.

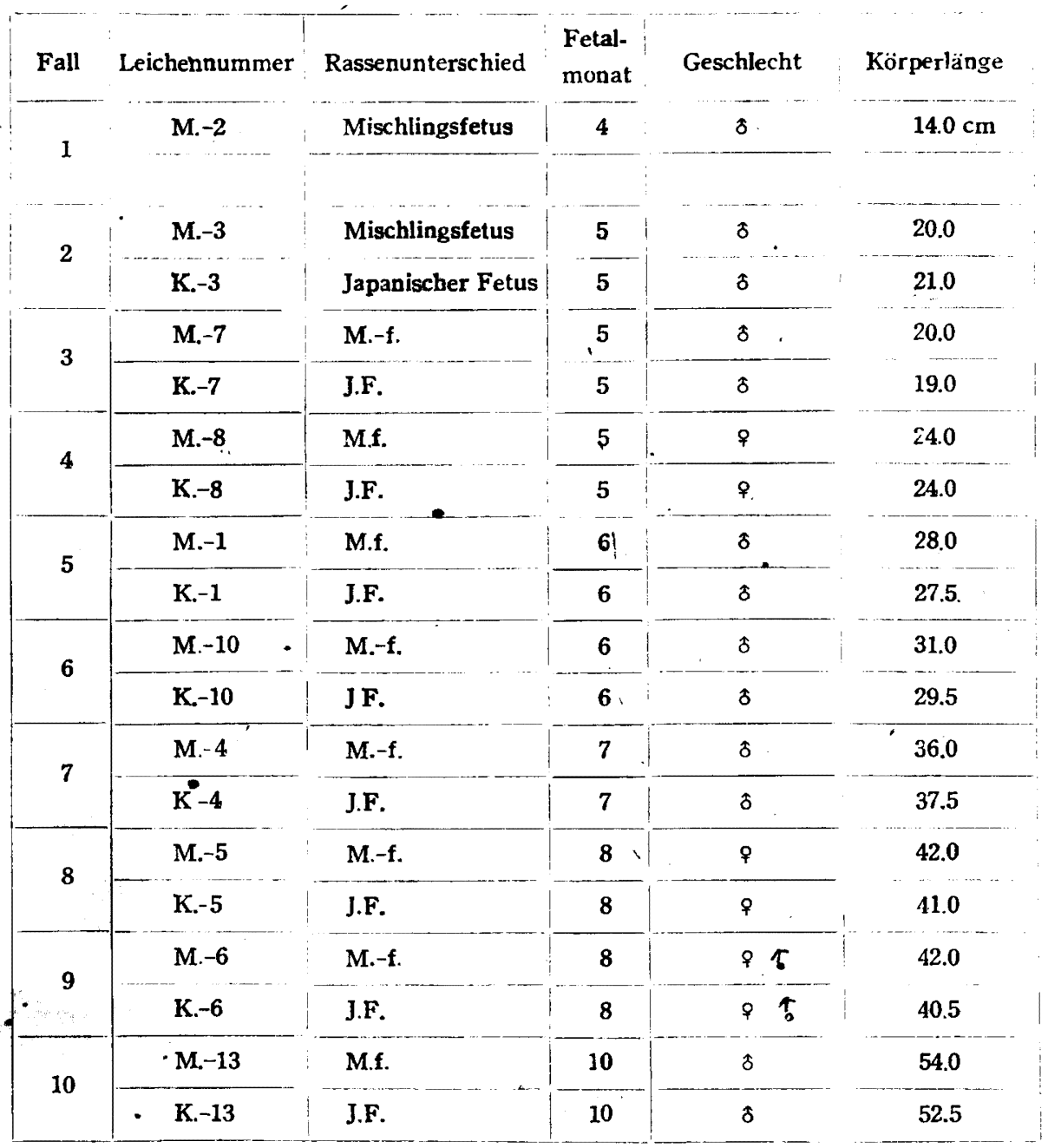

Fall 1, M.-2 Fall 8, M. 5: Mischlingsfeten zwischen Japanerinnen und Europäern. Fall 9, M.-6: Mischlingsfetus zwischen Japanerin und Neger.

Fall 10, M.-13: Mischlingsneugeborene zwischen Japanerin und Europäer. 
Es wurden aus den verschiedenen Körperteilen der Mischlinge, wie z. B. Stirn, Achselhöhle, Beugeseite des Oberarms, Handrücken, Kreuzgegend, Streckseite des Unterschenkels, Hautstücke entnommen, an denen nach der Einbettung in Paraffin und Herstellung von $10 \mu$ Schnitten eine schwache Färbung mit Hämatoxylin-Eosin durchgeführt wurde, Auch wurden, wo es nötig erschien, ungefärbte Präparate hergestellt, und bei denen, wo das Pigment schwer zu erkennen war, wurde die Versilberungsmethode angewendet., Dies geschah in der Weise, indem ich den Paraffinschnitten die 1\%-2\%ige Silbernitratlösung einwirken lie $B$, an denen nach $12-24$ stündiger Aufbewahrung im Ofen $\left(37^{\circ} \mathrm{C}\right)$, dann Spülung, darauf Einbringung in gesättigte Natrium-thiosulfatlösung und abermaliger Waschung die Kernfärbung mit Hämatoxylin durchgeführt wurde. Dabei traten die Pigmentkörner dunkel auf, zeigen sich mehr schwärzlich als schwarz-bräunlich, sehr deutlich, was die Mikroskopierung leicht ermöglichte.

\section{Eigene Befunde und Zusammenstellung.}

Seit früher her ist bekannt, daß im Hautpigment bei der Epidermis die zwischen den Epithelzellen vorhandenen Dendritenzellen (od. Langerhans' sche Zellen) und die Pigmentkörner erhaltenden Epithelzellen nachgewiesen sind: Prof. Adachi hat beim Corium zwei Arten von Zellen nachgewiesen: in der tieferen Schicht größere, und in der oberflächlicheren Schicht kleinere Pigmentzellen.

Ich habe in meiner Untersuchung des obigen Hautpigments bei Mischlingen und japanischen Feten von gleichem Fetalmonats die gleichen Körperteile verglichen und bin dabei zur folgenden Feststellung gelangt.

1. Dendritenzellen. Diese Zellen, welche Langerhans (1868) entdeckt hat, nahm er als Nervenzellen an. Sie weisen feine, verästelte Fortsätze auf und sind mit schwarz-bräunlichen Granula angefüllt, die erst in der intermediären Schicht auftreten und allmählich mit dem Fortschreiten des Fetallebens in der Basalschicht zahlreich zu sehen sind. Bisweilen kommen sie in der Grenze zwischen der Epidermis und dem Corium vor, indem sie an der Seite des Corium austretend liegen. Diese Pigmentzellen sind bei europäischen Feten nicht nach. weisbar (Adachi, Bahrawy), während ich sie bei japanischen Feten wie auch bei Mischlingsfeten beobachten konnte.

Ich habe beim Vergleich der Mischlingsfeten mit den zur Kontrolle herangezogenen japanischen Feten die Zahl der Dendritenzellen in einem 
Gesichtsfeld (im Okular, 10x Object, 40) den Durchschnittswert in 30 Gesichtsfeldern gesucht. Der Zahlenwert in der Tabelle 2, dessen Durchschnittswert , gering “ ist, versteht sich, wo in mehreren Gesichtsfeldern eine Dendritenzelle zu finden ist.

Tabelle 2. Zahl der Dendritenzellen an den verschiedenen Körperteilen.

\begin{tabular}{|c|c|c|c|c|c|c|c|c|c|}
\hline Fall & $\begin{array}{l}\text { Fetal- } \\
\text { monat }\end{array}$ & $\begin{array}{c}\text { Rassen- } \\
\text { unterschied }\end{array}$ & Stirn & $\begin{array}{l}\text { Achsel. } \\
\text { höhle }\end{array}$ & $\begin{array}{l}\text { Beuge- } \\
\text { seite d. } \\
\text { Ober- } \\
\text { armes }\end{array}$ & $\begin{array}{l}\text { Hand- } \\
\text { rücken }\end{array}$ & Rücken & $\begin{array}{l}\text { Kreuz- } \\
\text { gegend }\end{array}$ & $\begin{array}{l}\text { Streckseite } \\
\text { d. Unter- } \\
\text { schenkels }\end{array}$ \\
\hline 1 & $4 \mathrm{M}$ & $\begin{array}{l}\text { Mischlings- } \\
\text { fetus }\end{array}$ & 0 & 0 & 0 & & 0 & gering & \\
\hline \multirow{2}{*}{2} & \multirow{2}{*}{$5 \mathrm{M}$} & $\begin{array}{l}\text { Mischlings- } \\
\text { fetus }\end{array}$ & 2 & 0 & 0 & 1 & 0 & 0 & 1 \\
\hline & & $\begin{array}{l}\text { Japanischer } \\
\text { Fetus }\end{array}$ & 1 & 0 & 0 & 1 & 1 & 1 & 2 \\
\hline \multirow{2}{*}{3} & \multirow{2}{*}{$5 \mathrm{M}$. } & M.-f. & gering & 0 & 0 & 0 & 0 & 0 & gering \\
\hline & & J.F. & gering & 1 & gering & 0 & 2 & 0 & gering \\
\hline \multirow{2}{*}{4} & \multirow{2}{*}{$5 \mathrm{M}$. } & M. $-f$. & gering & 2 & gering & gering & 2 & 3 & 4 \\
\hline & & J.F. & gering & $Q$ & gering & gering & 0 & 3 & 5 \\
\hline \multirow{2}{*}{5} & \multirow{2}{*}{$6 \mathrm{M}$. } & M -f. & 0 & 0 & 0 & gering & gering & 0 & 1. \\
\hline & & J.F. & gering & 0 & 0 & gering & 2 & 0 & 2 \\
\hline \multirow{2}{*}{6} & \multirow{2}{*}{$6 \mathrm{M}$. } & M.-f. & 2 & 1 & gering & 2 & 1 & 1 & 3 \\
\hline & & J.F. & 0 & 0 & 1 & 2 & 6 & 6 & 5 \\
\hline \multirow{2}{*}{7} & \multirow{2}{*}{$7 \mathrm{M}$. } & M.-f. & 2 & gering & gering & 5 & gering & 2 & 3 \\
\hline & & J.F. & 2 & gering & gering & 2 & 5 & 3 & 5 \\
\hline \multirow{2}{*}{8} & \multirow{2}{*}{$8 \mathrm{M}$. } & M.-f. & 5 & 3 & 3 & 3 & 2 & 2 & 2 \\
\hline & & J.F. & 12 & 2 & 2 & 2 & 3 & 4 & 3 \\
\hline \multirow{2}{*}{9} & \multirow{2}{*}{$8 \mathrm{M}$. } & M.-f. & 9 & 15 & 5 & 8 & 10 & 11 & 9 \\
\hline & & J.F. & 5 & 1 & 1 & 1 & 2 & 5 & 6 \\
\hline \multirow{2}{*}{10} & \multirow{2}{*}{ 10M. } & M.-f. & 3 & 8 & 3 & 2 & 12 & 6 & 6 \\
\hline & & J.F. & 2 & 5 & 3 & gering & 5 & 5 & 3 \\
\hline
\end{tabular}


Wie die Tabelle 2. es zeigt, sind die Körperteile, wo die Dendritenzellen häufig vorkommen, bei japanischen Feten wie auch bei Mischlingsfeten Stirn, Rücken, Kreuzgegend und Streckseite des Unterschenkels. Die Körperteile, wo sie wenig zu finden sind, sind Achselhöhle, Beugeseite des Oberarms und Handrücken; diese Befunde stimmen meistens bei den verschiedenen Feten überein. Man kann auch einen mehr oder weniger Unterschied nach dem Körperteil nachweisen, aber im großen und ganzen scheint es, da $B$ die Dendritenzellen bei den japanischen Feten häufiger als bei Mischlingsfeten $z$ wischen Japanerin und Europäer sind.

Indes sind bei dem Mischlingsneugeborenen zwischen Europäer die Dendritenzellen häufiger als bei den zur Kontrolle herangezogenen 10 monatigem japanischen Fetus (s. Tabelle 2, Fall 10). Auch bei dem Mischlingsfetus von Neger sind diese Pigmentzellen auffallend häufig zu sehen (s. Tabelle 2, Fall 9).

2. Intrazelluläre Pigmentkörner der Epidermis. Die feinen schwarzbräunlichen Granula sind in der Basalschicht häufig, nämlich über das ganze Basalepithel diffus, oder stellenweise gruppiert vorhanden. Man kann die Pigmentkörner, wo sie reichlich vorkommen, im oberen Teil des Kerns kappenartig, auch bis in die obere mehrere Schicht der Basalschicht, und beim Mischlingsfetus $z$ wischen Neger auch bis in die Hornschicht verfolgen. Wenn man diese Pigmentkörner, wie es die Tabelle 3 zeigt, mit reichliche $(H)$, mittlere $(H)$, geringe $(+)$, äußerst geringe $( \pm)$ und Fehlen $(-)$ darstellt, so stimmt die Menge der Pigmentkörner bei Mischlingsfeten wie auch japanischen Feten meist regionär überein, und wenn man es zusammenfassend betrachtet, so scheint es, daß bei japanischen Feten die reichlichere Neigung zu Pigmentkörnern vorliegt. Indes verhält es sich bei Mischlingsneugeborenen ähnlich wie bei den Dendritenzellen, indem man die Vermehrung der Pigmentkörner in den verschiedenen Körperteilen deutlicher als beim Embryo beobachtet. (s. Tabelle 3, Fall 10). Beim Mischlingsfetus $z$ wischen Neger sind die Pigmentkörner in den verschiedenen Körperteilen häufiger als bei denen der Kontrolle. (s. Tabelle 3, Fall 9).

3. Größere Pigmentzellen im Corium. Diese Pigmentzellen sind hauptsächlich oval, spindelförmig, auch bandförmig und enthalten reichliche Menge von schwarz-bräunlichen Granula in Größe von 3050-80 $\mu$.

Nach Adachi werden die Pigmentzellen bei Europäern nach der Geburt beobachtet. Dahingegen sah sie Bahrawy bei der Durchführung der Versilberungsmethode bereits im 5 . Fetalmonat. In meinem 
Tabelle 3. Intrazelluläre Pigmentkörner an den verschiedenen Körperteilen.

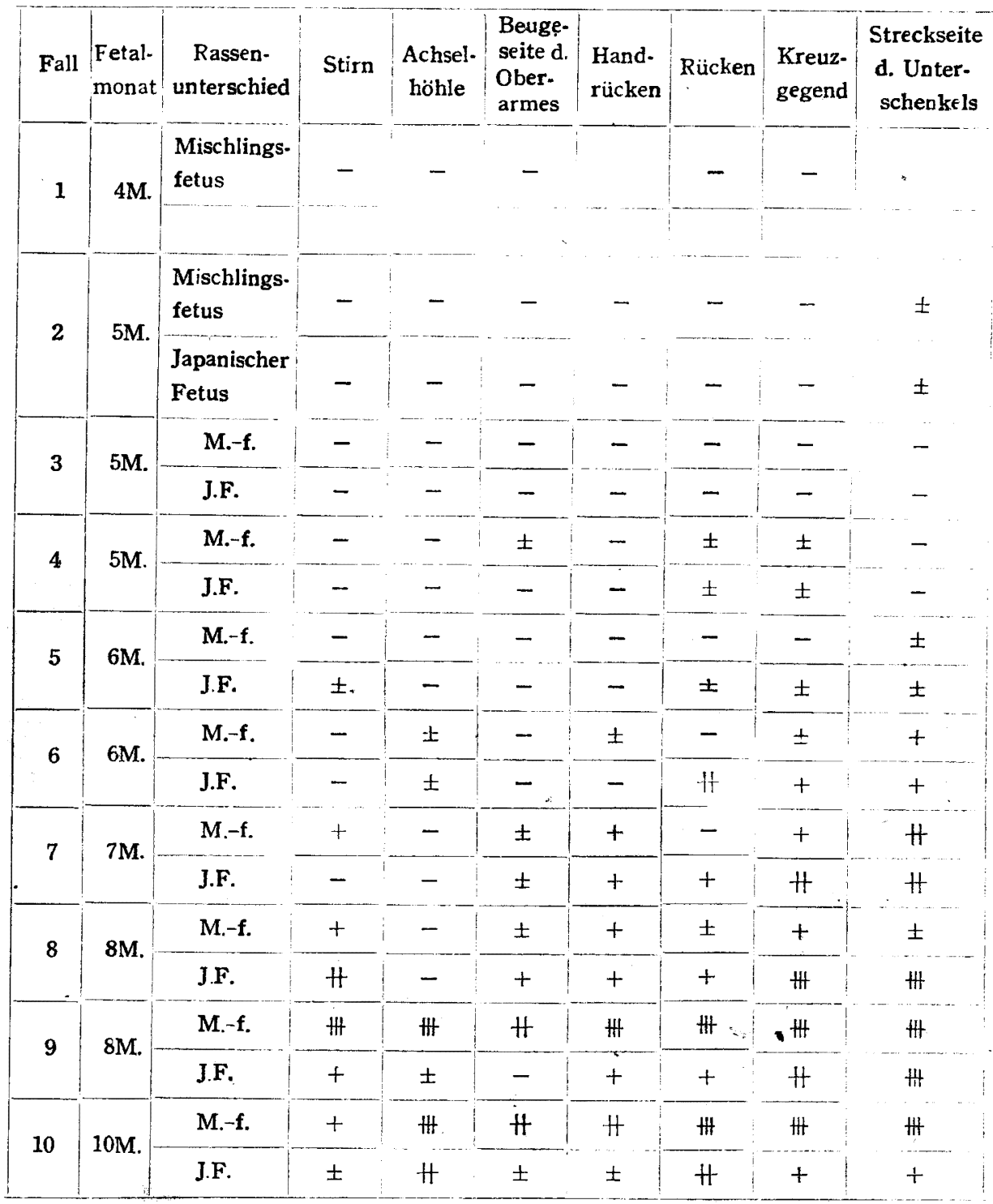

Material sind sie bei japanischen Feten in der Kreuzgegend in allen Fällen zu sehen. Bei Mischlingsfeten, ausgenommen 2 Fälle (Mischlingsfeten $z$ wischen Europäern), konnte ich die Anhäufung von größeren Pigmentzellen im unteren Zweidrittel der tieferen Schicht des Corium 
manchmal sogar im subcutanen Fettgewebe beobachten. Diese Zellen verursachen das sogenannte Mongolenfleck. Jedoch sind diese größeren

Tabelle 4. Zahl der größeren Pigmentzellen an der Kreuzgegend.

\begin{tabular}{|c|c|c|c|}
\hline Fall & Fetalmonat & Mischlingsfeten & Japanische Feten \\
\cline { 2 - 4 } 1 & $4 \mathrm{M}$. & gering & \\
\hline 2 & $5 \mathrm{M}$. & 0 & 4 \\
\hline 3 & $5 \mathrm{M}$. & 2 & 3 \\
\hline 4 & $5 \mathrm{M}$. & gering & gering \\
\hline 5 & $6 \mathrm{M}$. & 0 & 6 \\
\hline 6 & $6 \mathrm{M}$. & 6 & 7 \\
\hline 7 & $7 \mathrm{M}$. & 3 & 28 \\
\hline 8 & $8 \mathrm{M}$. & 4 & 26 \\
\hline 9 & $8 \mathrm{M}$. & 15 & 16 \\
\hline 10 & $10 \mathrm{M}$. & 6 & 15 \\
\hline
\end{tabular}

Fig. 1. Kreuzhaut des Mischlingsfetus (Fall. 8. 40 × 10).

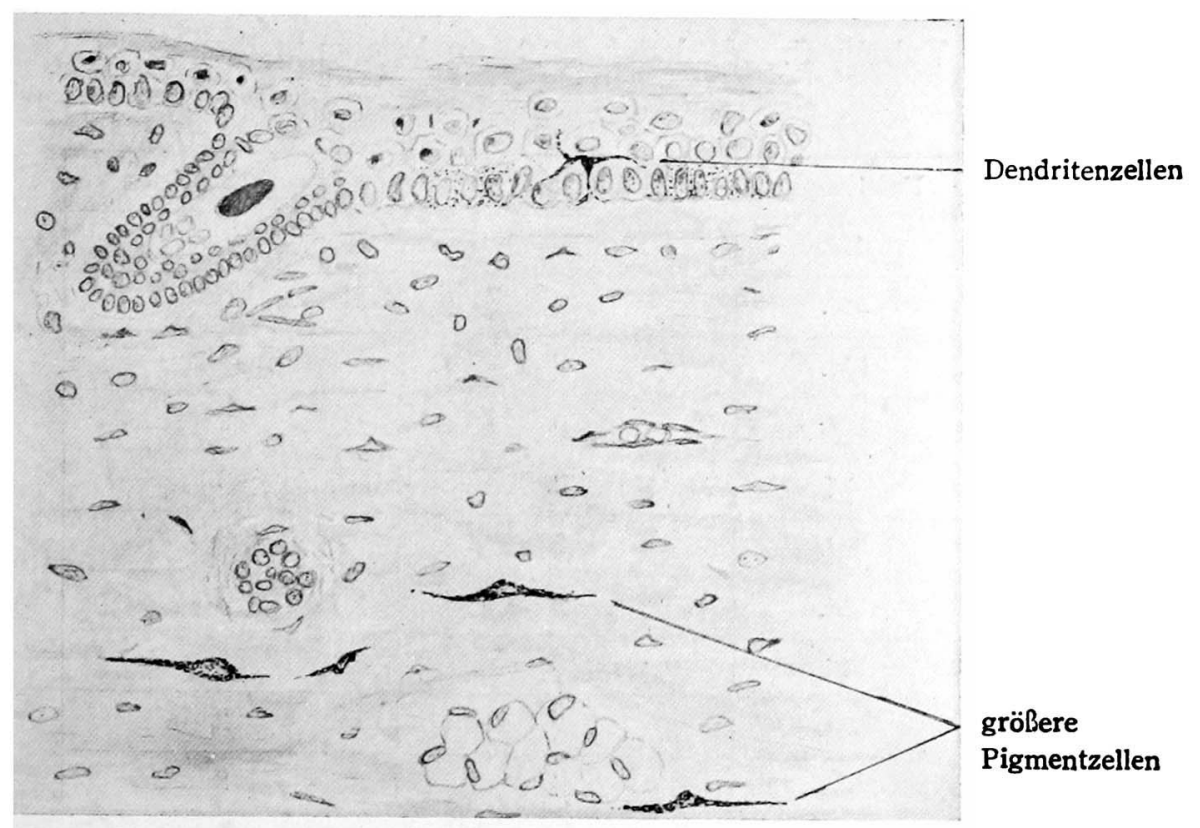


Pigmentzellen, wie die Tabelle 4 es zeigt, bei Mischlingsfeten zwischen Europäern in 5 von 8 Fällen, geringer an Zahl als bei den Kontroll-

Fig. 2. Kreuzhaut des japanischen Fetus (Fall 8, $40 \times 10$ ).

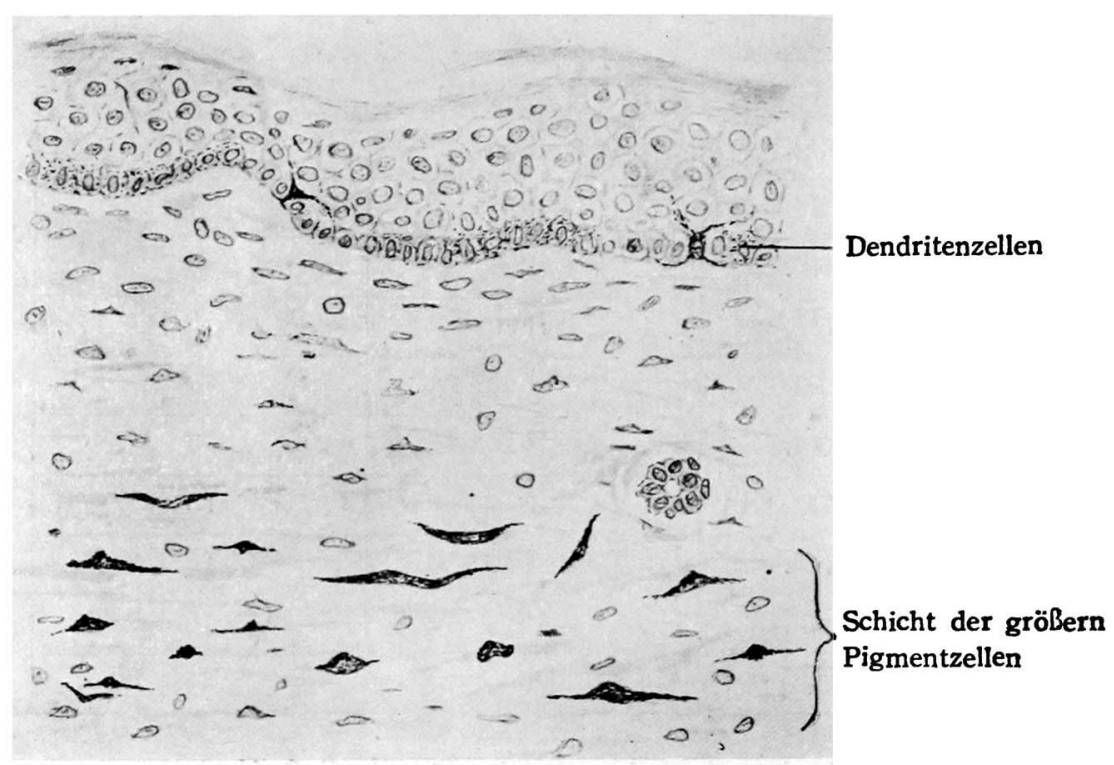

Tabelle 5. Zahl der größeren Pigmentzellen an dem Handrücken.

\begin{tabular}{|c|c|c|c|}
\hline Fall & Fetalmonat & Mischlingsfeten & Japanische Feten \\
\hline 1 & $4 \mathrm{M}$ & & \\
\hline 2 & $5 \mathrm{M}$. & 2 & 2 \\
\hline 3 & $5 \mathrm{M}$ & 2 & gering \\
\hline 4 & $5 \mathrm{M}$. & 0 & 2 \\
\hline 5 & $6 \mathrm{M}$. & 2 & 3 \\
\hline 6 & $6 \mathrm{M}$. & 2 & 2 \\
\hline 7 & $7 \mathrm{M}$. & $\mathbf{0}$ & 4 \\
\hline 8 & $8 \mathrm{M}$. & 3 & 5 \\
\hline 9 & $8 \mathrm{M}$. & 6 & 2 \\
\hline 10 & $10 \mathrm{M}$. & 4 & 2 \\
\hline
\end{tabular}


fällen, und bei Mischlingsfeten von Neger ist ein quantitativer Unterschied nicht zu beobachten.

Außer der Kreuzhaut treten die größeren Pigmentzellen reichlich auf im Handrücken und Streckseite des Unterschenkels, sie bilden jedoch, wie in der Kreuzhaut, gruppenweise und keine Schicht. Eine besondere interessante Tatsache ist, $\mathrm{da} B$ im Handrücken die japanischen Feten in allen Fällen, die Mischlingsfeten mit Ausnahme von 2 Fällen alle in einem Gesichtsfeld 2-6 Pigmentzellen aufweisen. Ihre Zahl ist bei weitem geringer als in der Kreuzhaut, und scheint bei Mischlingsfeten von Europäern etwas geringer als bei den zur Kontrolle herangezogenen japanischen Feten zu sein. Es ließ sich jedoch wie in der Kreuzhaut ein quantitativer Unterschied $z$ wischen beiden Feten nicht deutlich nachweisen.

Die oberflächlichere Schicht des Corium, nahe der Basalschicht des Epidermis vorhandenen sogenannten kleineren Pigmentzellen, ließ sich in allen Fällen nicht beobabhten. Man sagt, da $\beta$ diese Pigmentzellen im Fetalleben selten auftreten.

\section{Schluss.}

1) Es wird angeführt, da $B$ bei europäischen Feten das Epidermispigment nicht zu beobachten ist (Adachi, Bahrawy), jedoch konnte ich sie bei Mischlingsfeten zwischen Japanerinnen und Europäern bei Feten des 5. Fetallebens bereits sehen. Das Epidermispigment der Mischlingsfeten $z$ wischen Japanerinnen und Europäern ist geringer an Menge als bei den japanischen Feten des gleichen Fetalmonats.

2) Der Mischlingsfetus zwischen Japanerin und Neger weist deutlich reichlichere Menge des Epidermispigments auf als der japanische Fetus des gleichen Fetalmonats.

3) Obwohl bei Mischlingsfeten zwischen Japanerinnen und Europäern das Epidermispigment geringer ist als bei japanischen Feten des gleichen Fetalmonats, kann man beim Mischlingsneugeborenen von Europäer eine deutlichere Vermehrung des Pigments als bei dem japanischen Fetus des 10. Fetalmonats nachweisen. Hieraus kann man folgern, da $B$ das Epidermispigment des Fetus, sobald er den Mutterleib verlässt, rasch zuzunehmen scheint.

4) Bei Mischlingsfeten wie auch bei japanischen Feten sind die Körperteile, wo das Epidermispigment reichlich ist, Rücken, Kreuzgegend und Streckseite des Unterschenkels, und wo es wenig vorkommt, die Beugeseite des Oberarms. Also, wir erkennen bei beiden eine 
Übereinstimmung in der Verteilung des Epidermispigments.

5) Die größeren Pigmentzellen im Corium werden bei den Mischlingsfeten beobachtet. Uud in der Kreuzgegend bei japanischen Feten sind diese Pigmentzellen reichlicher an Zahl als bei Mischlingsfeten zwischen Japanerinnen und Europäern.

Am schlusse dieser Arbeit spreche ich Herrn Prof. Tanig uchi fürdie unermüdliche Leitung in dieser Forschung meinen aufrichtigsten Dank aus. Auch danke ich Herrn Dr. Mitsui für cen Beistand, ebenfalls Herrn Dr. Wat a nabe für die liebenswürdige Überlassung des Unterschungsmaterials.

\section{Literatur.}

v) Adachi, B, Hautpigment beim Menschen und den Affen. Zeitschr. f. Morph. u. Anthropol., Bd. 6. 1903.

$\checkmark 2)$ Adachi, B, u. Fujisawa, K., Mongolenfleck bei Europäern, Zeitschr. f. Morph. u. Anthropol. Bd. 6. 1903.

${ }^{3)}$ Bahrawy, El., Über das Mongolenfleck bei Europäern. Ein Beitrag zur Pigmentlehre. Arch. f. Derm. u. Syph. Bd. 141. 1922.

4) Caspary, J., Über den Ort der Bildung des Hautpigmentes. Arch. f. Derm. u. Syph. Bd. 23. 1891 .

5) Fischel, A., Zur Pigmentenwicklung. Anat. Anz. Bd. 2. 1896.

6) Grimm, F., Beiträge zum Stadium des Pigments. Derm. Zeitschr. Bd 2. 1895.

7) Hasegawa, ., Über das Spitzenpigment bei den Japanern. Kaibogaku.Zasshi. Bd. 2. 1929. (Japanisch).

8) Hoepke, H., Die Haut. Handbuch der mikroskopischen Anatomie des Menschen. (W. v, Möllendorff) 1927.

9) Iizuka, H., Untersuchung über das Pigment der unteren Extremitaten bei den Japanern. Kaibogaku-Zasshi. Bd. 4. 1931. (Japanisch).

10) Ishikawa, N. Über das sogenannte Mongolenfleck bei japanischen Feten. Folia Anat. Japon. Bd. 2. 1924.

11) Katsunuma, S., Pigmentzellen in der Pia mater bei Japanern und ihre Beziehung zur Geschwulst. „Gann“ (Japan. Zeitschr. f. Krebsforschung) Bd. XI. Heft 4. 1917. (Japanisch).

12) Morooka, K., Über das Mongolenfleck bei Japanern. Kaibogaku-Zasshi. Bd. 3. 1931. (Japanisch).

13) Muto, C. u. Miwa, T., Über das Vorkommen des Hautpigments des Fetus bei Japanern. Chosenigakukai-Zasshi. Bd. 50. 1924. (Japanisch).

14) Takeda, R., Zwei Fälle von Mongolenfleck. Kaibogaku-Zasshi. Bd. 5. 1932. (Japanisch).

15) Yamano, K., Genetische Untersuchung des Gesichtshautpigments bei Japanern. Kaibogaku-Zasshi. Bd. 3. 1930. (Japınisch).

16) Kudo, T., Mischlingsfeten zwischen Japanerinnen und Europäer und Negern Igakuto-Seibutsugaku. Bd. 10. 1947. (Japanisch). 